\title{
EXPANDING METRICS FOR UNICRITICAL SEMIHYPERBOLIC POLYNOMIALS
}

\author{
LUKAS GEYER
}

\begin{abstract}
We prove that unicritical polynomials $f(z)=z^{d}+c$ which are semihyperbolic, i.e., for which the critical point 0 is a non-recurrent point in the Julia set, are uniformly expanding on the Julia set with respect to the metric $\rho(z)|d z|$, where $\rho(z)=1+\operatorname{dist}(z, P(f))^{-1+1 / d}$ has singularities on the postcritical set $P(f)$. We also show that this metric is Hölder equivalent to the usual Euclidean metric.
\end{abstract}

\section{Contents}

1. Introduction

1.1. Notation and background

1.2. Results and questions on expanding metrics

1

2. Distortion estimates, hyperbolic and orbifold metrics 4

3. John domains, Gehring trees and singular metrics 6

4. Semihyperbolicity 9

5. Unicritical polynomials 10

References 13

\section{INTRODUCTION}

In the theory of dynamics of polynomials and rational functions of degree at least 2 in one complex dimension, by far the best understood class is that of hyperbolic maps. Various weaker notions of hyperbolicity have also been introduced and investigated. In order to state definitions, results, and questions, let us introduce some fairly standard notation. For background in complex dynamics, see one of the many excellent textbooks, e.g., CG93] or [Mil06].

1.1. Notation and background. For a rational map $f: \hat{\mathbb{C}} \rightarrow \hat{\mathbb{C}}$ of degree $d \geq 2$ we denote its forward iterates by $f^{n}$, its set of critical points by $\operatorname{Crit}(f)$, its postcritical set by $P(f)=$ $\overline{\bigcup_{n=1}^{\infty} f^{n}(\text { Crit } f)}$, and its Julia set by $J(f)$. A periodic cycle of period $q \geq 1$ is a $q$-tuple of distinct points $Z=\left(z_{0}, z_{1}, \ldots, z_{q-1}\right)$ with $f\left(z_{k}\right)=z_{k+1}$ and $f\left(z_{q-1}\right)=z_{0}$. Its multiplier is $\lambda=\left(f^{q}\right)^{\prime}\left(z_{0}\right)=\left(f^{q}\right)^{\prime}\left(z_{k}\right)$. Since the multiplier is invariant under Möbius conjugacy, it is also defined for cycles containing $\infty$. A periodic cycle $Z$ with multiplier $\lambda$ is attracting if $|\lambda|<1$, repelling if $|\lambda|>1$, and indifferent if $|\lambda|=1$. An indifferent cycle is rationally indifferent (or parabolic) if $\lambda$ is a root of unity, irrationally indifferent otherwise. A point $z$ is attracted to the cycle $Z$ if $\operatorname{dist}\left(f^{n}(z), Z\right) \rightarrow 0$ as $n \rightarrow \infty$. The $\omega$-limit set $\omega(z)=\omega_{f}(z)$ of a point $z$ under $f$ is the set of all $\operatorname{limits}_{\lim _{k \rightarrow \infty}} f^{n_{k}}(z)$ for sequences $n_{k} \rightarrow \infty$. A point $z \in \hat{\mathbb{C}}$ is non-recurrent if it has a neighborhood which does not contain any of its forward iterates $f^{n}(z)$ for $n \geq 1$, or equivalently if $z \notin \omega(z)$. For polynomials we denote the basin of infinity by $A(\infty)=A_{f}(\infty)$.

2010 Mathematics Subject Classification. Primary 37F15; Secondary 30C20, 30F45. 
Definition 1. Let $f: \hat{\mathbb{C}} \rightarrow \hat{\mathbb{C}}$ be a rational function of degree $d \geq 2$. Then $f$ is

- hyperbolic if each critical points of $f$ is attracted by an attracting periodic cycle;

- subhyperbolic if each critical point in the Fatou set of $f$ is attracted by an attracting periodic cycle, and each critical point in the Julia set of $f$ is strictly preperiodic;

- semihyperbolic if each critical point in the Fatou set of $f$ is attracted by an attracting periodic cycle, and each critical point in the Julia set of $f$ is non-recurrent.

It is obvious that hyperbolic $\Longrightarrow$ subhyperbolic $\Longrightarrow$ semihyperbolic. We are particularly interested in the question how these notions are related to expansion on the Julia set $J(f)$, in the following sense:

Definition 2. Let $f: \hat{\mathbb{C}} \rightarrow \hat{\mathbb{C}}$ be a rational function of degree $d \geq 2$ with $\infty \notin J(f)$, let $U$ be an open connected neighborhood of $J(f)$, and let $\rho: U \rightarrow(0,+\infty]$ be a continuous function. We denote by $d_{\rho}$ the induced path metric on $U$, defined by

$$
d_{\rho}\left(z_{0}, z_{1}\right)=\inf \left\{\int_{\gamma} \rho(z)|d z|: \gamma \subseteq U \text { piecewise smooth curve from } z_{0} \text { to } z_{1}\right\} .
$$

Then

(1) $f$ is expanding with respect to $\rho(z)|d z|$ if there exist $C>0$ and $\lambda>1$ such that

$$
\frac{\left|\left(f^{n}\right)^{\prime}(z)\right| \rho\left(f^{n}(z)\right)}{\rho(z)} \geq C \lambda^{n}
$$

for a dense set of $z \in J(f)$ and all $n \geq 1$.

(2) $\rho(z)|d z|$ induces the standard topology (or is topologically equivalent to the Euclidean metric) on $J(f)$ if the identity map from $\left(J(f), d_{\rho}\right)$ to $J(f)$ equipped with the standard Euclidean metric is a homeomorphism.

(3) $\rho(z)|d z|$ is Hölder equivalent to the Euclidean metric on $J(f)$ if the identity map from $\left(J(f), d_{\rho}\right)$ to $J(f)$ equipped with the standard Euclidean metric is bi-Hölder, i.e., it is Hölder continuous with Hölder continuous inverse.

Remark. The assumption that $\infty \notin J(f)$ is only added for convenience. In the case where $\infty \in J(f)$, one should replace the Euclidean metric with the spherical metric. Note that $d_{\rho}\left(z_{0}, z_{1}\right)$ might a priori be infinite.

The following results are known, see e.g. [CG93, Ch. V] or [Mil06, § 19].

Theorem 3. A rational map $f$ with $\infty \notin J(f)$ is hyperbolic iff it is expanding with respect to the standard Euclidean metric. It is subhyperbolic iff it is expanding with respect to a metric $\rho(z)|d z|$ whose metric density $\rho(z)$ is a continuous function in an open connected neighborhood $U$ of $J(f)$ except for finitely many singularities $a_{1}, \ldots, a_{q}$, such that there exists $C>0$ and $\beta<1$ with

$$
C^{-1} \leq \rho(z) \leq \sum_{k=1} \frac{C}{\left|z-a_{k}\right|^{\beta}}
$$

for all $z \in U \backslash\left\{a_{1}, \ldots, a_{q}\right\}$.

Remark. In the case where $\infty \in J(f)$, a similar result with the spherical metric instead of the Euclidean metric is still true. In the subhyperbolic case, any metric $\rho(z)|d z|$ where $\rho$ satisfies (11) is Hölder equivalent to the Euclidean metric, so in particular it induces the same topology. 
1.2. Results and questions on expanding metrics. If one allows infinitely many singularities of the metric $\rho(z)|d z|$, the following is a natural question.

Question 4. Which rational maps $f$ admit expanding metrics $\rho(z)|d z|$ topologically equivalent (or Hölder equivalent) to the spherical metric (or Euclidean metric in the case $\infty \notin J(f))$ ?

Remark. It is probably easier to first ask this only for polynomials. As far as the author knows, the answer is not known even in the quadratic family $f_{c}(z)=z^{2}+c$.

In CJY94, p. 9, Remark 2], Carleson, Jones, and Yoccoz claim that an expanding metric can easily be constructed for semihyperbolic polynomials. However, they only give a formula for the case of quadratic polynomials, and they do not provide a proof either of expansion or of the fact that this metric is topologically equivalent to the Euclidean metric. Later, in his $\mathrm{Ph}$. D. thesis [Car97, Carette provided a proof of expansion with respect to the metric $\operatorname{dist}(z, P(f))^{-1 / 2}|d z|$ in the case of quadratic polynomials, combining expansion on the postcritical set given by a result of Mãne from [Mañ93] with expansion in the complement of the postcritical set with respect to its hyperbolic metric. However, Carette's thesis was never published, and the part of his proof that the singular metric induces the standard Euclidean topology on the Julia set is flawed.

The goal of this paper is to fill in this gap in the literature in the case of unicritical polynomials $f(z)=z^{d}+c$, combining the results of Mañé, Carleson, Jones, and Yoccoz with basic distortion theorems and estimates of the hyperbolic and orbifold metric of punctured disks. In particular, we are not combining two different kinds of expansion like Carette did, which in the opinion of the author makes this proof conceptually simpler. The proof that the singular metric is topologically (in fact, Hölder) equivalent to the Euclidean metric uses the fact that the basin of infinity is a John domain, which was proved in [CJY94].

Generalizing the proof to semihyperbolic polynomials with more than one critical point seems to be non-trivial, for at least a couple of different reasons: It is possible that the $\omega$-limit set of one critical point contains another critical point, so the different parts of the postcritical sets might not be "separated", and one might not have expansion on the postcritical set with respect to the Euclidean metric. Furthermore, the argument given in this paper for the fact that the singular metric $\rho(z)|d z|$ induces the same topology on the Julia set crucially uses that there are no bounded Fatou components. Semihyperbolic polynomials with more than one critical point can have bounded attracting domains, in which case our proof does not work.

Whether expanding metrics exist for semihyperbolic rational functions, or for polynomials satisfying weaker conditions such as Collet-Eckmann are very interesting question which warrant further investigation. In order to allow for metrics $\rho$ with singularities on all of $J(f)$, the definition of expansion might have to be modified for these cases.

Our main result is the following, proved in Section 5 .

Theorem 13. Assume that the polynomial $f(z)=z^{d}+c$ is semihyperbolic, but not hyperbolic. Then $f$ is expanding with respect to the metric $\rho(z)|d z|$, where $\rho(z)=1+\frac{1}{\operatorname{dist}(z, P(f))^{1-1 / d}}$. Explicitly, there exist $C>0$ and $\lambda>1$ such that

$$
\left|\left(f^{n}\right)^{\prime}(z)\right| \rho\left(f^{n}(z)\right) \geq C \lambda^{n} \rho(z)
$$

for all $z \in J(f) \backslash P(f)$ and all $n \geq 1$. Furthermore, the metric $\rho(z)|d z|$ is Hölder equivalent to the Euclidean metric on $\mathbb{C}$.

Remark. The neighborhood of the Julia set here is $U=\mathbb{C}$, so we consider the induced path metric $d_{\rho}$ on the whole plane. Note that the postcritical set $P(f)$ is a forward-invariant compact proper subset of the Julia set, which implies that it is nowhere dense in $J(f)$.

In the case of subhyperbolic polynomials, the metric $\rho(z)|d z|$ is a metric satisfying the conditions of Theorem 3 with $\beta=1-1 / d$, and it is in fact bi-Lipschitz equivalent in a neighborhood 
of the Julia set to the canonical orbifold metric associated to $f$. For a definition of the canonical orbifold metric and its use in the proof of expansion in the subhyperbolic case, see [CG93, Ch. V] or [Mil06, § 19]. It seems that semihyperbolic polynomials which are not subhyperbolic do not have a useful associated orbifold structure, since $P(f) \cap J(f)$ is not a discrete set in that case.

\section{Distortion estimates, Hyperbolic And orbifold Metrics}

We will use the notation $B\left(z_{0}, r\right)=\left\{z \in \mathbb{C}:\left|z-z_{0}\right|<r\right\}, \bar{B}\left(z_{0}, r\right)=\overline{B\left(z_{0}, r\right)}$, and $S\left(z_{0}, r\right)=$ $\partial B\left(z_{0}, r\right)=\left\{z \in \mathbb{C}:\left|z-z_{0}\right|=r\right\}$ for the open and closed disk, and the circle of radius $r>0$ centered at $z_{0} \in \mathbb{C}$, respectively.

Koebe's distortion theorems are usually stated for normalized conformal maps, see e.g. Dur83. The following versions for general conformal maps in disks are immediate consequences by translation and rescaling.

Theorem 5 (Koebe Distortion and 1/4-Theorem). Let $g: B\left(z_{0}, r\right) \rightarrow \mathbb{C}$ be univalent with $w_{0}=g\left(z_{0}\right)$. Then

$$
\frac{\left|g^{\prime}\left(z_{0}\right)\right|}{\left(1+\frac{\left|z-z_{0}\right|}{r}\right)^{2}} \leq\left|\frac{g(z)-g\left(z_{0}\right)}{z-z_{0}}\right| \leq \frac{\left|g^{\prime}\left(z_{0}\right)\right|}{\left(1-\frac{\left|z-z_{0}\right|}{r}\right)^{2}}
$$

for all $z \in B\left(z_{0}, r\right) \backslash\left\{z_{0}\right\}$. Furthermore, the disk $B\left(w_{0}, \frac{\left|g^{\prime}\left(z_{0}\right)\right| r}{4}\right)$ is contained in the image $g\left(B\left(z_{0}, r\right)\right)$.

We will frequently apply this theorem to inverse branches of iterates of $f$. We define the conformal radius $r\left(U, z_{0}\right)$ of a simply connected domain $U \subsetneq \mathbb{C}$ with respect to a point $z_{0} \in U$ as the unique $r>0$ for which there is a conformal map $g: B(0, r) \rightarrow U$ with $g(0)=z_{0}$ and $g^{\prime}(0)=1$. If we denote the hyperbolic metric of $U$ by $\delta_{U}(z)|d z|$, then conformal invariance of the hyperbolic metric and the explicit formula $\delta_{B(0, r)}=\frac{2 r}{r^{2}-|z|^{2}}$ immediately give the well-known relation

$$
\delta_{U}\left(z_{0}\right)=\frac{2}{r\left(U, z_{0}\right)}
$$

between conformal radius and density of the hyperbolic metric. Furthermore, by the Schwarz lemma we know that $U$ can not be contained in any disk $B\left(z_{0}, r^{\prime}\right)$ with $r^{\prime}<r$, so that

$$
\operatorname{diam} U \geq r\left(U, z_{0}\right) \text {. }
$$

It also immediately follows from the definition that the conformal radius of the image of $U$ under a conformal map $g: U \rightarrow V$ with respect to the point $w_{0}=g\left(z_{0}\right)$ is

$$
r\left(V, w_{0}\right)=\left|g^{\prime}\left(z_{0}\right)\right| r\left(U, z_{0}\right) .
$$

An orbifold is a topological space locally modeled on the quotient of Euclidean space by a finite group. In the context of complex analysis, we are mostly interested in Riemann orbifolds (as a generalization of Riemann surfaces) which are locally modeled on the unit disk $\mathbb{D}$ or on the quotient of the unit disk by the group $\Gamma_{d}$ of rotations of $d$-th roots of unity. For background on Riemann orbifolds see McM94.

A point on the orbifold which corresponds to the fixed point 0 of $\Gamma_{d}$ in such a chart is called a cone point of order $d$. If $\phi: U \rightarrow \mathbb{D} / \Gamma_{d}$ is such a chart, then $\phi_{d}: U \rightarrow \mathbb{D}, \phi_{d}(z)=\phi(z)^{d}$ is a homeomorphism. If we replace each chart $\phi$ by $\phi_{d}$, we get an atlas of a Riemann surface, the underlying Riemann surface $\mathcal{S}$ of the orbifold $\mathcal{O}$. This also shows that every Riemann orbifold can be specified by giving a Riemann surface $\mathcal{S}$ and a discrete set of cone points $z_{k}$ with 
corresponding orders $d_{k}=d\left(z_{k}\right) \geq 2$. A map $f: \mathcal{O} \rightarrow \tilde{\mathcal{O}}$ between orbifolds is an analytic orbifold map if $f: \mathcal{S} \rightarrow \tilde{\mathcal{S}}$ is an analytic map between the underlying Riemann surfaces satisfying

$$
d(f(z)) \mid d(z) \operatorname{deg}(f, z)
$$

for all $z \in \mathcal{O}$, where $d(z)$ and $d(f(z))$ are the orders of the cone points at $z$ and $f(z)$, respectively, with the convention that $d(z)=1$ for points which are not cone points. It is an analytic orbifold covering map if it is an analytic branched covering from $\mathcal{S}$ to $\tilde{\mathcal{S}}$, with

$$
d(f(z))=d(z) \operatorname{deg}(f, z)
$$

for all $z \in \mathcal{O}$. It is known [McM94, Theorem A.2] that the only Riemann orbifolds which do not have a Riemann surface cover are the teardrop ( $\hat{\mathbb{C}}$ with one cone point of order $d \geq 2$ ) and the spindle ( $\hat{\mathbb{C}}$ with two cone points of orders $d_{1}, d_{2} \geq 2$ with $d_{1} \neq d_{2}$ ). All other Riemann orbifolds have as universal cover either the sphere, the plane, or the disk, respectively called elliptic, parabolic, or hyperbolic. A Riemann orbifold $\mathcal{O}$ with underlying Riemann surface $\mathcal{S}$ is hyperbolic iff its Euler characteristic

$$
\chi(\mathcal{O})=\chi(\mathcal{S})-\sum_{k}\left(1-\frac{1}{d\left(z_{k}\right)}\right)
$$

is negative. This shows that most orbifolds are hyperbolic, and that in particular every orbifold whose underlying Riemann surface is hyperbolic is itself hyperbolic. If $\mathcal{O}$ is a hyperbolic Riemann orbifold, and if $\phi: \mathbb{D} \rightarrow \mathcal{O}$ is an analytic orbifold covering map, then $\mathcal{O}$ inherits a hyperbolic metric from pushing forward the hyperbolic metric $\frac{2|d z|}{1-|z|^{2}}$ on the unit disk, and since any two such analytic orbifold covering map differ by composition with a hyperbolic isometry, this metric is independent of the choice of $\phi$. We call this metric the hyperbolic orbifold metric of $\mathcal{O}$ and denote it by $\delta_{\mathcal{O}}(z)|d z|$, and the induced hyperbolic orbifold distance between points $z, w \in \mathcal{O}$ by $\delta_{\mathcal{O}}(z, w)$. As a special case, if $\mathcal{O}=\mathcal{S}$ is a Riemann surface, the hyperbolic orbifold metric $\delta_{\mathcal{S}}(z)|d z|$ is just the hyperbolic metric of $\mathcal{S}$. For convenience, we will also introduce the pseudohyperbolic orbifold distance $p_{\mathcal{O}}(z, w)$, defined in the unit disk by

$$
p_{\mathbb{D}}(z, w)=\left|\frac{z-w}{1-\bar{w} z}\right|,
$$

and pushed forward by the analytic orbifold covering $\phi: \mathbb{D} \rightarrow \mathcal{O}$ as usual. The pseudo-hyperbolic distance is a metric, but it is not generated by a path metric and it is not complete if $\mathcal{O}$ is not compact. The hyperbolic and pseudo-hyperbolic distances are related by

$$
\delta_{\mathcal{O}}(z, w)=2 \tanh ^{-1} p_{\mathcal{O}}(z, w)=\log \frac{1+p_{\mathcal{O}}(z, w)}{1-p_{\mathcal{O}}(z, w)} .
$$

The next rather elementary, but very useful lemma compares the hyperbolic metric and the hyperbolic orbifold metric in the case of simply connected domains in the plane with a single cone point.

Theorem 6. Let $U \subsetneq \mathbb{C}$ be a simply connected domain, let $z_{0} \in U, d \geq 2$, and let $\mathcal{O}$ be the Riemann orbifold with underlying domain $U$ and a single cone point of order $d$ at $z_{0}$, Then

$$
\frac{\delta_{\mathcal{O}}(z)}{\delta_{U}(z)}=F_{d}\left(p_{U}\left(z_{0}, z\right)\right) \quad \text { for } z \in U \backslash\left\{z_{0}\right\}
$$

with

$$
F_{d}(t)=\frac{1-t^{2}}{d t^{1-1 / d}\left(1-t^{2 / d}\right)}=\frac{t^{-1}-t}{d\left(t^{-1 / d}-t^{1 / d}\right)} \quad \text { for } 0<t<1
$$


Furthermore, we have the estimates

$$
\frac{1}{d} \leq F_{d}(t) t^{1-1 / d} \leq 1 \quad \text { for } 0<t<1
$$

Proof. Let $\psi: U \rightarrow \mathbb{D}$ be a conformal map from the underlying domain $U$ to $\mathbb{D}$ with $\psi\left(z_{0}\right)=0$. Then $\psi$ is also an orbifold covering map of the orbifold $\mathcal{O}$ to the orbifold $\mathcal{O}_{d}$ with underlying domain $\mathbb{D}$ and a cone point of order $d$ at 0 . Analytic orbifold covering maps lift to hyperbolic isometries, so they are local isometries with respect to the respective hyperbolic orbifold metrics, i.e., $\delta_{\mathcal{O}_{d}}(\psi(z))\left|\psi^{\prime}(z)\right|=\delta_{\mathcal{O}}(z)$ for all $z \in \mathcal{O}$. The same is true for the hyperbolic metrics of the underlying domains, i.e., $\delta_{\mathbb{D}}(\psi(z))\left|\psi^{\prime}(z)\right|=\delta_{U}(z)$ for all $z \in U$. This implies that

$$
\frac{\delta_{\mathcal{O}}(z)}{\delta_{U}(z)}=\frac{\delta_{\mathcal{O}_{d}}(\psi(z))}{\delta_{\mathbb{D}}(\psi(z))}
$$

for all $z \in U$. Since conformal maps of simply connected domains are isometries with respect to the hyperbolic and pseudo-hyperbolic metrics, we also get that $p_{U}\left(z_{0}, z\right)=p_{\mathbb{D}}(0, \psi(z))$, so it is enough to prove the theorem for the case of $U=\mathbb{D}, z_{0}=0$, and $\mathcal{O}=\mathcal{O}_{d}$. In this case a hyperbolic orbifold covering $\phi: \mathbb{D} \rightarrow \mathcal{O}$ is given by $\phi(z)=z^{d}$, so the hyperbolic orbifold metric of $\mathcal{O}_{d}$ is given by

$$
\delta_{\mathcal{O}_{d}}(z)=\frac{2}{d|z|^{1-1 / d}\left(1-|z|^{2 / d}\right)}
$$

This shows that

$$
\frac{\delta_{\mathcal{O}_{d}}(z)}{\delta_{\mathbb{D}}(z)}=\frac{1-|z|^{2}}{d|z|^{1-1 / d}\left(1-|z|^{2 / d}\right)}=\frac{|z|^{-1}-|z|}{d\left(|z|^{-1 / d}-|z|^{1 / d}\right)}=F_{d}(|z|)
$$

for all $z \in \mathbb{D}$, and since $|z|=p_{\mathbb{D}}(0, z)$ the first claim in the theorem follows. The claimed estimates follow easily from the explicit calculation

$$
F_{d}(t) t^{1-1 / d}=\frac{1-t^{2}}{d\left(1-t^{2 / d}\right)}=\frac{1+t^{2 / d}+t^{4 / d}+\ldots+t^{(2 d-2) / d}}{d} .
$$

\section{John domains, Gehring trees and singular metrics}

Roughly speaking, a John domain is a domain in which one can connect any two points without getting too close to the boundary. This concept was introduced in the context of elasticity by John in [Joh61], and the term "John domain" was coined by Martio and Sarvas in [MS79]. By now, John domains have found many applications in various branches of geometric analysis, and there are multiple different equivalent definitions. For an excellent in-depth survey and the comparison of various definitions for both bounded and unbounded domains see [NV91]. There are some subtleties in the case of unbounded domains, where the various different common definitions of John domains are not always equivalent. However, the difficulties only appear in the case where $\infty$ is a boundary point (when viewing these domains as subsets of the Riemann sphere), whereas in the setting of our paper we always have that $G \subset \hat{\mathbb{C}}$ is the basin of infinity for a polynomial, so $\infty$ is an interior point of $G$.

Definition 7. A domain $G \subsetneq \hat{\mathbb{C}}$ with $\infty \notin \partial G$ is a John domain if there exists a constant $c \in(0,1)$ such that the following holds: For every $z_{0}, z_{1} \in G$ there exists a simple path $\gamma \subset G$ from $z_{0}$ to $z_{1}$ in $G$ such that

$$
\operatorname{dist}(z, \partial G) \geq c \min \left\{l\left(\gamma\left(z_{0}, z\right)\right), l\left(\gamma\left(z, z_{1}\right)\right)\right\}
$$


for all $z \in \gamma$, where $\gamma\left(z_{0}, z\right)$ and $\gamma\left(z, z_{1}\right)$ are the sub-paths from $z_{0}$ to $z$ and from $z$ to $z_{1}$, respectively, and where $l($.$) denotes Euclidean arc length. Any such path is called a John path$ (or John arc in the case where $\gamma$ is an arc) from $z_{0}$ to $z_{1}$.

Note that we allow $z_{0}$ or $z_{1}$ to be infinite, in which case the minimum on the right is the length of the bounded sub-path.

Since we are only dealing with unicritical semihyperbolic polynomials, there are no bounded Fatou components, so the Julia set $J=\partial G=\hat{\mathbb{C}} \backslash G$ is a continuum with empty interior which does not disconnect the plane. Following [LR18, we call such a continuum $J \subset \mathbb{C}$ whose complement $G=\hat{\mathbb{C}} \backslash J$ is a John domain, a Gehring tree. By the Riemann mapping theorem there exists a conformal map $\phi: \Delta \rightarrow G$, where $\Delta=\hat{\mathbb{C}} \backslash \overline{\mathbb{D}}$. For convenience we choose the unique such map for which $\phi(\infty)=\infty$ and $\lim _{z \rightarrow \infty} \frac{\phi(z)}{z} \in(0, \infty)$. Since boundaries of John domains are locally connected, $\phi$ extends continuously to the closure $\bar{\Delta}$. Following the conventions in complex dynamics, we will call the parametrized curve $\gamma(r)=\phi\left(r e^{i \theta}\right), 1 \leq r \leq \infty$, the external ray of angle $\theta$. Note that external rays are hyperbolic geodesics.

By [NV91, Theorem 5.2], see also [GHM89, Theorem 4.1], in the case of simply connected hyperbolic domains, hyperbolic geodesics are John arcs with some constant $c^{\prime} \in(0,1)$ which only depends on the John constant $c$. It is clear by a simple limit argument that hyperbolic geodesics terminating at a point in $\partial G$ still satisfy the John condition. In the rest of this section we adopt the convention that $c \in(0,1)$ is a John constant which in particular works for all hyperbolic geodesics, our "canonical" John arcs.

Theorem 8. Let $J \subset \mathbb{C}$ be a Gehring tree, $\alpha \in(0,1)$ be a constant, and let $\rho(z)=1+\frac{1}{\operatorname{dist}(z, J)^{\alpha}}$. Then the path metric in $\mathbb{C}$ defined by $\rho(z)|d z|$ is Hölder equivalent to the Euclidean metric

In the context of semihyperbolic polynomials, the metric is defined slightly differently, where the Julia set $J$ in the definition of the metric is replaced by the postcritical set $P$. However, the same result follows easily.

Corollary 9. Let $J \subset \mathbb{C}$ be a Gehring tree, $P \subset J$ be a compact non-empty set, $\alpha \in(0,1)$ be a constant, and let $\rho(z)=1+\frac{1}{\operatorname{dist}(z, P)^{\alpha}}$. Then the path metric in $\mathbb{C}$ defined by $\rho(z)|d z|$ is Hölder equivalent to the Euclidean metric.

Proof of Corollary 9. This is a simple "sandwiching" argument. Let us denote the two metrics by $\rho_{P}$ and $\rho_{J}$. We have that $|d z| \leq \rho_{P}(z)|d z| \leq \rho_{J}(z)|d z|$, and since $\rho_{J}(z)|d z|$ is Hölder equivalent to the Euclidean metric by Theorem 8 , the same is true for $\rho_{P}(z)|d z|$.

Proof of Theorem 8 . We will actually show that

$$
\left|z_{0}-z_{1}\right| \leq d_{\rho}\left(z_{0}, z_{1}\right) \leq C\left|z_{0}-z_{1}\right|^{1-\alpha}
$$

for all $z_{0}, z_{1} \in \mathbb{C}$ with $\left|z_{0}-z_{1}\right|<1$. The lower estimate follows directly from $\rho(z) \geq 1$, so let us prove the upper estimate. For convenience, we will write $\delta(z)=\operatorname{dist}(z, \partial G)$. In this proof, $C_{1}, C_{2}, \ldots$ will denote positive constants which depend only on $\alpha$ and the John constant $c$. The sketch in Figure 1 should help illuminate the argument.

Let us first compare Euclidean and $\rho$-length of John arcs. Assume that $\gamma:[0, L] \rightarrow \bar{G}$ is an arc length parametrization of a hyperbolic geodesic between $z_{0}=\gamma(0)$ and $z_{1}=\gamma(L)$, where we allow $z_{0}$ or $z_{1}$ to be on the boundary of $G$. Let $\gamma_{0}=\left.\gamma\right|_{[0, L / 2]}$ be the first half of this geodesic segment and $\gamma_{1}=\left.\gamma\right|_{[L / 2, L]}$ the second half. Then

$$
l_{\rho}\left(\gamma_{0}\right)-\frac{L}{2}=\int_{\gamma_{0}} \frac{|d z|}{\delta(z)^{\alpha}}=\int_{0}^{L / 2} \frac{d t}{\delta(\gamma(t))} \leq \int_{0}^{L / 2} \frac{d t}{(c t)^{\alpha}}=\frac{L^{1-\alpha}}{(1-\alpha) 2^{\alpha} c^{\alpha}}
$$




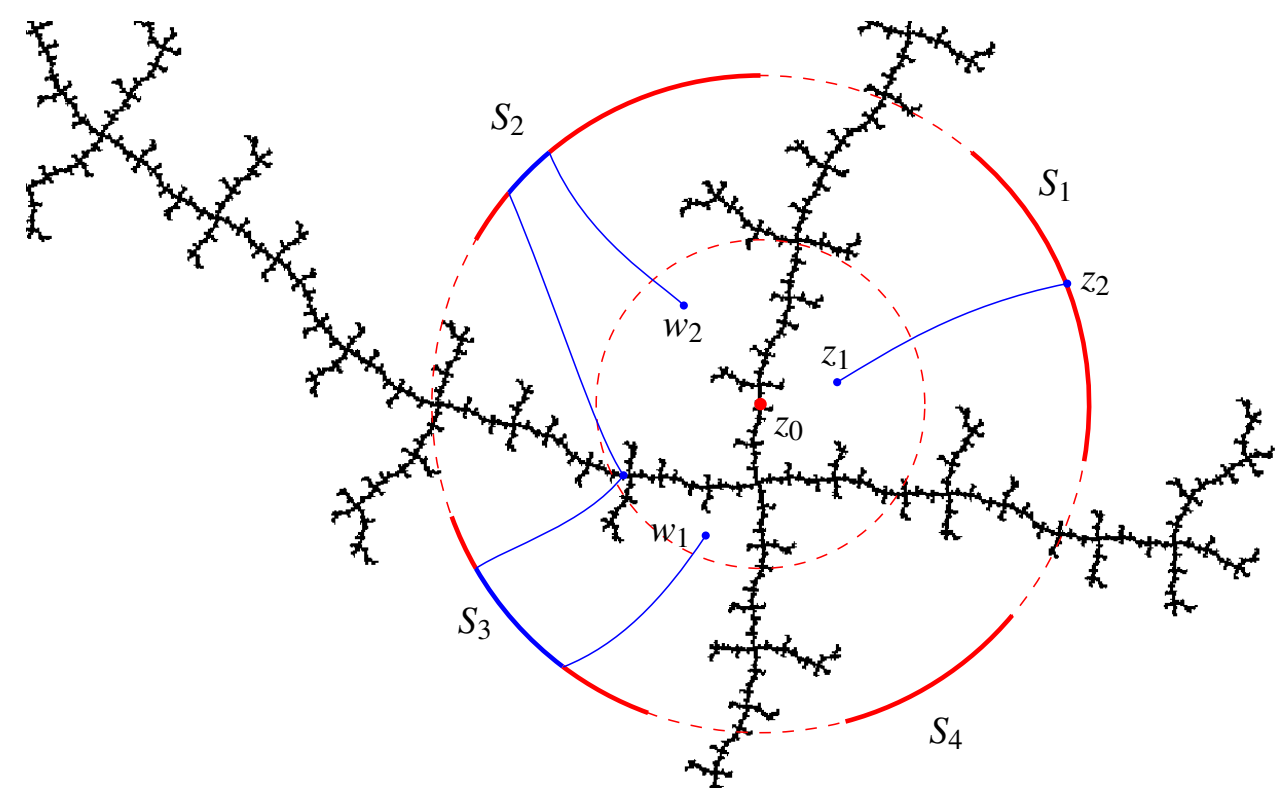

FiguRE 1. Sketch of the construction in the proof for an example of a Gehring tree, part of the subhyperbolic Julia set of $f(z)=z^{2}+c$ with $c \approx 0.419643+0.606291 i$ (produced with Curt McMullen's software from http://math.harvard.edu/ ctm/programs/index.html). Dashed circles are $S\left(z_{0}, r\right)$ and $S\left(z_{0}, 2 r\right)$, solid circular arcs are $S_{k} \subseteq S\left(z_{0}, 2 r\right)$. Part of an external ray is drawn from $z_{1}$ to $z_{2}$. All external rays from $\bar{B}\left(z_{0}, r\right)$ exit $B\left(z_{0}, 2 r\right)$ through one of the arcs $S_{k}$. The number of arcs, the $\rho$-length of the arcs and the parts of external rays contained in $\left|z-z_{0}\right| \leq 2 r$ are all controlled by the John constant and $r$. The path between $w_{1}$ and $w_{2}$ illustrates how any two points in the $r$-neighborhood can be connected in $\bar{B}\left(z_{0}, 2 r\right)$ by a controlled number of external rays and subarcs of $S_{k}$, each of controlled length, connecting through points in $B\left(z_{0}, r\right) \cap J$.

and the same estimate holds for $l_{\rho}\left(\gamma_{1}\right)$, so that

$$
l_{\rho}(\gamma) \leq l(\gamma)+C_{1} l(\gamma)^{1-\alpha}
$$

This estimate is already sufficient to show that $d_{\rho}$ is Hölder equivalent to the internal Euclidean metric of $G$. However, two points $z_{0}, z_{1} \in G$ with $\left|z_{0}-z_{1}\right|$ small can have a large internal Euclidean distance. (As an example, if $J=[-1,1]$, and $z_{0}=\epsilon i, z_{1}=-\epsilon i$, we have $\left|z_{0}-z_{1}\right|=2 \epsilon$, whereas the internal distance in $G=\mathbb{C} \backslash[-1,1]$ is always $\geq 2$.)

As a next step we will find an upper bound for the $\rho$-diameter of Euclidean disks centered at points in $J$. Fix $z_{0} \in J$ and $r \in(0,1)$. Consider an arbitrary $z_{1} \in \bar{B}\left(z_{0}, r\right)$ and the external ray $\gamma$ from $z_{1}$ up to its first intersection with $S\left(z_{0}, 2 r\right)$ at some point $z_{2}$. (In the case where $z_{1} \in \partial G$ is the landing point of more than one ray, let $\gamma$ be one of these rays.) Then $l(\gamma) \geq\left|z_{2}-z_{1}\right| \geq r$, so by the John property $B\left(z_{2}, c r\right) \subseteq G$. On the other hand, we know from the John property that $l(\gamma) \leq \frac{2 r}{c}$, since $\delta\left(z_{2}\right) \leq 2 r$, so by (9) we have that

$$
l_{\rho}(\gamma) \leq \frac{2 r}{c}+C_{1}\left(\frac{2 r}{c}\right)^{1-\alpha} \leq \frac{2 r^{1-\alpha}}{c}+C_{1}\left(\frac{2 r}{c}\right)^{1-\alpha}=C_{2} r^{1-\alpha}
$$

The set

$$
K=\left\{z \in S\left(z_{0}, 2 r\right): \delta(z) \geq c r\right\}
$$


is compact, and so it can be covered by finitely many disks of radius $\mathrm{cr} / 2$ centered at points in $K$. The union of these disks has finitely many connected components $U_{1}, \ldots, U_{N}$, and their intersection with the circle $S\left(z_{0}, 2 r\right)$ consists of finitely many disjoint circular $\operatorname{arcs} S_{1}, \ldots, S_{N}$, each with an arc length of at least $c r$, so that $N \leq 4 \pi / c$, an upper bound depending only on the John constant $c$. We also have that

$$
l_{\rho}\left(S_{j}\right)=l\left(S_{j}\right)+\int_{S_{j}} \frac{|d z|}{\delta(z)^{\alpha}} \leq\left[1+\left(\frac{2}{c r}\right)^{\alpha}\right] l\left(S_{j}\right) \leq\left[\frac{1}{r^{\alpha}}+\frac{2^{\alpha}}{c^{\alpha} r^{\alpha}}\right] 4 \pi r=C_{3} r^{1-\alpha} .
$$

Now define $B_{j}$ to be the set of points $z_{1} \in \bar{B}\left(z_{0}, r\right)$ for which an external ray ${ }^{1}$ from $z_{1}$ intersects $\overline{U_{j}}$. Since every such ray intersects $K$, and $K \subset \bigcup_{j=1}^{N} U_{j}$, we have that $\bar{B}\left(z_{0}, r\right)=\bigcup_{j=1}^{N} B_{j}$. Any two points $z_{1}, z_{2} \in B_{j}$ can be joined by a path $\gamma$ in $\bar{B}\left(z_{0}, 2 r\right)$ by joining the external rays from $z_{1}$ and $z_{2}$ to $S_{j}$ with an arc of $S_{j}$, of total $\rho$-length $l_{\rho}(\gamma) \leq C_{4} r^{1-\alpha}$, by (10) and (11), so that $\operatorname{diam}_{\rho} B_{j} \leq C_{4} r^{1-\alpha}$. Since $\bar{B}\left(z_{0}, r\right)=\bigcup_{j=1}^{N} B_{j}$ is a connected union we have that $\operatorname{diam}_{\rho} \bar{B}\left(z_{0}, r\right) \leq C_{5} r^{1-\alpha}$ with $C_{5}=N C_{4}$.

Now fix any $z_{0}, z_{1} \in \mathbb{C}$ with $r=\left|z_{0}-z_{1}\right| \in(0,1)$. Then one of the following is true.

Case 1: There exists $z_{2} \in \bar{B}\left(z_{0}, 2 r\right) \cap J$.

In this case, $\bar{B}\left(z_{0}, r\right) \subset \bar{B}\left(z_{2}, 3 r\right)$, so $\operatorname{diam}_{\rho} B\left(z_{0}, r\right) \leq \operatorname{diam}_{\rho} B\left(z_{2}, 3 r\right) \leq C_{6} r^{1-\alpha}$.

Case 2: $\bar{B}\left(z_{0}, 2 r\right) \cap J=\emptyset$.

In this case $\delta(z) \geq r$ for every $z \in \bar{B}\left(z_{0}, r\right)$, so if $\gamma \subset \bar{B}\left(z_{0}, r\right)$ is a straight line segment, then $l_{\rho}(\gamma) \leq l(\gamma)\left(1+r^{-\alpha}\right) \leq 4 r^{1-\alpha}$, so $\operatorname{diam}_{\rho} B\left(z_{0}, r\right) \leq 4 r^{1-\alpha}$.

These cases combined show in particular that $d_{\rho}\left(z_{0}, z_{1}\right) \leq C\left|z_{0}-z_{1}\right|^{\alpha}$ with $C=\max \left\{C_{6}, 4\right\}$, proving (8) and finishing the proof.

\section{Semihyperbolicity}

Recall from the introduction that a polynomial $f$ is semihyperbolic if each critical point in the Fatou set of $f$ is attracted by an attracting periodic cycle, and each critical point in the Julia set of $f$ is non-recurrent. Following ideas of Mañé from [Mañ93], Carleson, Jones, and Yoccoz in CJY94 showed the following.

Theorem 10 (Carleson, Jones, Yoccoz). Let $f$ be a polynomial of degree $\geq 2$. Then the following are equivalent:

(1) $f$ is semihyperbolic.

(2) There exists $\epsilon>0, C>0, \theta \in(0,1)$, and $D \in \mathbb{N}$ such that for all $x \in J(f)$, for all $n \in \mathbb{N}$, and for all components $U$ of $f^{-n}(B(x, \epsilon))$, we have that $\operatorname{diam} U \leq C \theta^{n}$, and that $\operatorname{deg}\left(f^{n}, U\right) \leq D$.

(3) $A_{\infty}(f)$ is a John domain.

(4) $A_{\infty}(f)$ is a John domain and every bounded Fatou domain is a quasidisk.

Let us say that a backward orbit of a domain $U \subset \hat{\mathbb{C}}$ is a sequence of domains $\left(U_{n}\right)$ such that $U_{0}=U$, and $U_{n}$ is a connected component of $f^{-1}\left(U_{n-1}\right)$ for $n \geq 1$. In particular this implies that the restriction $\left.f\right|_{U_{n}}: U_{n} \rightarrow U_{n-1}$ is a branched covering for every $n \geq 1$. The sequence $\left(d_{n}\right)$ defined by $d_{n}=\left.\operatorname{deg} f\right|_{U_{n}}$ for $n \geq 1$ is the sequence of degrees of this backward orbit. Notice that $\left.f^{n}\right|_{U_{n}}: U_{n} \rightarrow U$ is a branched covering of degree $\prod_{k=1}^{n} d_{k}$. We say that a backward orbit $\left(U_{n}\right)$ is univalent if $d_{n}=1$ for all $n$.

The following theorem is a slightly refined version of Theorem 2.1 in [CJY94.

\footnotetext{
${ }^{1}$ For $z_{1} \in G$ there is a unique external ray, but we allow $z_{1} \in \partial G$, in which case there might be several.
} 
Theorem 11. Let $f$ be a polynomial of degree $d \geq 2$ without parabolic periodic points and without recurrent critical points. Let $c_{1}, \ldots c_{r}$ be the distinct critical points of $f$, of multiplicities $m_{1}, \ldots, m_{r}$, and let $\epsilon_{0}>0$ be arbitrary. Then there exists $\epsilon>0, C_{0}>0$, and $\theta \in(0,1)$ such that the following holds: For all $z \in J(f)$, and for any backward orbit $\left(U_{n}\right)$ of $U=B(z, \epsilon)$ we have

(1) $\operatorname{diam} U_{n} \leq \min \left\{C_{0} \theta^{n}, \epsilon_{0}\right\}$.

(2) Each $U_{n}$ contains at most one critical point, and each critical point $c_{j}$ is contained in at most one $U_{n}$.

(3) Each $U_{n}$ is simply connected, and the sequence of degrees $\left(d_{n}\right)$ satisfies $d_{n}=1$ if $U_{n}$ does not contain a critical point, and $d_{n}=m_{j}+1$ if $c_{j} \in U_{n}$.

(4) The degree of $\left.f^{n}\right|_{U_{n}}: U_{n} \rightarrow U$ is bounded by $D=\prod_{j=1}^{r}\left(m_{j}+1\right) \leq 2^{d-1}$.

Proof. Essentially all these properties directly follow from the proof in [CJY94, but we will show how all of these follow from their stated results as well as the results from [Mañ93]. Property (1) combines results from these two papers with a compactness argument. Properties (3) and (4) are immediate consequences of (2), which is the main point of this refinement. We may assume by possibly choosing $\epsilon_{0}$ smaller that for every critical point $c \in J(f)$ the $2 \epsilon_{0}$-neighborhood $B\left(c, 2 \epsilon_{0}\right)$ does not contain any other critical point, and no point of the forward orbit of $c$. Since $f$ has no parabolic periodic points and no recurrent critical points, by [Mañ93, Theorem II (a)], for every $z \in J(f)$ there exists a neighborhood $U_{z}$ of $z$, so that every connected component of $f^{-n}\left(U_{z}\right)$ has diameter $<\epsilon_{0}$. Then $\left\{U_{z}\right\}_{z \in J(f)}$ is an open cover of the compact Julia set of $f$, so it has a finite subcover $\left\{U_{z_{k}}\right\}_{k=1}^{m}$, with some Lebesgue number $\epsilon>0$, i.e., such that for any $z_{0} \in J(f)$, the $\epsilon$-neighborhood $B\left(z_{0}, \epsilon\right)$ is contained in $U_{z_{k}}$ for some $1 \leq k \leq m$.

Now let $z \in J(f)$ be arbitrary, and let $\left(U_{n}\right)$ be a backward orbit of $U=B(z, \epsilon)$. Then $\operatorname{diam} U_{n}<\epsilon_{0}$ for all $n \geq 0$, and so by our assumption on $\epsilon_{0}$ each $U_{n}$ can contain at most one critical point. If some critical point $c$ is contained in $U_{n}$ and $U_{n+j}$ with $n, j \in \mathbb{N}, j \geq 1$, then $U_{n+j}$ contains both $c$ and $f^{j}(c)$, contradicting our assumption on $\epsilon_{0}$. Combined this shows (2).

The fact that there exist constants $C_{0}>0$ and $\theta \in(0,1)$ (independent of $z$ ) with diam $U_{n} \leq$ $C_{0} \theta^{n}$ for all $n \geq 0$ follows from [CJY94, Theorem 2.1], where we might have to allow for making $\epsilon$ smaller.

Preimages of simply connected domains under polynomials are simply connected, so all $U_{n}$ are simply connected. The degree of compositions of branched covers is the product of the degrees, and since each critical point appears at most once in the sequence $\left(U_{n}\right)$, property $(4)$ immediately follows.

\section{UNICRITICAL POLYNOMIALS}

Up to conjugation, unicritical polynomials are polynomials of the form $f(z)=z^{d}+c$ with $d \geq 2$, and critical point 0 of multiplicity $d-1$. Such a polynomial is semihyperbolic iff $0 \notin \omega(0)$. The main technical result in this section is Theorem 12, which shows that a semihyperbolic polynomial $f(z)=z^{d}+c$ is expanding with respect to the singular metric $\frac{|d z|}{\operatorname{dist}(z, P(f))^{1-1 / d}}$. Here is a rough sketch of the proof strategy: In the unicritical case, the statement of Theorem 11 takes a particularly simple form. Every backward orbit $\left(U_{n}\right)$ of a small disk $U$ is either univalent, or there is exactly one $n_{0}$ for which $d_{n_{0}}=d$, and $d_{n}=1$ for all $n \neq n_{0}$. In the case where $f^{n}$ does not have a critical point in $U_{n}$, it is a conformal map from $U_{n}$ to $U$, and thus a hyperbolic isometry. If $f^{n}$ has a critical point in $U_{n}$, then there is only one, and $f_{n}$ is a branched cover from $U_{n}$ to $U$ of degree $d$, with exactly one branch point $w_{n}$ of local degree $d$. Equivalently, $f^{n}$ is an orbifold covering map from $U_{n}$ to $\mathcal{O}$, where $\mathcal{O}$ is the orbifold with underlying domain $U$ and a single cone point of order $d$ at the critical value $w_{0}=f^{n}\left(w_{n}\right)$. Combining the exponential 
shrinking of the diameters of $U_{n}$ from Theorem 11 with Koebe distortion estimates and the comparison of orbifold and hyperbolic metrics from Section 2, we obtain the desired metric distortion estimates for $f$ with respect to the metric $\rho(z)|d z|$.

Theorem 12. Assume that the polynomial $f(z)=z^{d}+c$ is semihyperbolic but not hyperbolic. Then $f$ is expanding with respect to the metric $\sigma(z)|d z|$, where $\sigma(z)=\frac{1}{\operatorname{dist}(z, P(f))^{1-1 / d}}$. Explicitly, there exist $C>0$ and $\lambda>1$ such that

$$
\left|\left(f^{n}\right)^{\prime}(z)\right| \sigma\left(f^{n}(z)\right) \geq C \lambda^{n} \sigma(z)
$$

for all $z \in J(f) \backslash P(f)$ and all $n \geq 1$.

Proof. Let us first note that $P(f)$ is a forward-invariant compact proper subset of the Julia set $J(f)$, so it is in particular nowhere dense in $J(f)$. By establishing (12) for all $z \in J(f) \backslash P(f)$, we thereby show that it holds on a dense subset of the Julia set, which establishes that $f$ is expanding with respect to $\sigma$.

Fix constants $\epsilon>0, C_{0}>0$, and $\theta \in(0,1)$ from Theorem 11, We define $\lambda:=\theta^{-1 / d}>1$. In the course of this proof, $C_{1}, C_{2}, \ldots$ will denote positive constants which depend only on $\epsilon, C_{0}$, $\theta$, and $d$.

Pick an arbitrary $z_{0} \in J(f)$, and a backward orbit $\left(z_{n}\right)_{n=0}^{\infty}$ of $z_{0}$, i.e., a sequence satisfying $f\left(z_{n}\right)=z_{n-1}$. In order to prove the theorem, we will show that the inequality (12) holds for all $z_{n}$, as long as $z_{n} \notin P(f)$, with constants only depending on $\epsilon, C_{0}, \theta$, and $d$. Let $U_{0}=U=B\left(z_{0}, \epsilon\right)$, and let $U_{n}$ be the connected component of $f^{-n}(U)$ containing $z_{n}$. Let $r_{n}=r\left(U_{n}, z_{n}\right)$ be the conformal radius of $U_{n}$ with respect to $z_{n}$. By definition, Theorem 11, and inequality (44) we have that

$$
r_{0}=\epsilon \quad \text { and } \quad r_{n} \leq \operatorname{diam} U_{n} \leq C_{0} \theta^{n}
$$

for all $n \geq 1$. Now we fix some arbitrary $n$, assume that $z_{n} \notin P(f)$, and consider the following three cases separately

(1) $f^{n}$ maps $U_{n}$ conformally onto $U$, and $P(f) \cap U_{n}=\emptyset$.

(2) $f^{n}$ maps $U_{n}$ conformally onto $U$, and $P(f) \cap U_{n} \neq \emptyset$.

(3) $f^{n}$ has a critical point in $U_{n}$.

In cases (1) and (2) we know from (5) that

$$
\left|\left(f^{n}\right)^{\prime}\left(z_{n}\right)\right|=\frac{r_{0}}{r_{n}}
$$

Case 1: $f^{n}$ maps $U_{n}$ conformally onto $U$, and $P(f) \cap U_{n}=\emptyset$.

In this case Koebe's $1 / 4$-theorem shows that $\operatorname{dist}\left(z_{n}, P(f)\right) \geq \frac{r_{n}}{4}$, so that $\sigma\left(z_{n}\right) \leq\left(\frac{4}{r_{n}}\right)^{1-1 / d}$. We also observe that $m=\min _{z \in J(f)} \sigma(z)>0$, so $\sigma\left(z_{0}\right) \geq m$. Combining these estimates with (14), we get that

$$
\begin{aligned}
\frac{\left|\left(f^{n}\right)^{\prime}\left(z_{n}\right)\right| \sigma\left(z_{0}\right)}{\sigma\left(z_{n}\right)} & \geq m \frac{r_{0}}{r_{n}}\left(\frac{r_{n}}{4}\right)^{1-1 / d}=\frac{m \epsilon}{2 \cdot 4^{1-1 / d}} r_{n}^{-1 / d} \\
& \geq \frac{m \epsilon}{2 \cdot 4^{1-1 / d} C_{0}^{1 / d}}\left(\theta^{-1 / d}\right)^{n}=C_{1} \lambda^{n}
\end{aligned}
$$

Case 2: $f^{n}$ maps $U_{n}$ conformally onto $U$, and $P(f) \cap U_{n} \neq \emptyset$.

We are assuming $z_{n} \notin P(f)$, so there exists $w_{n} \in P(f) \cap U_{n}$ with $\operatorname{dist}\left(z_{n}, P(f)\right)=\left|z_{n}-w_{n}\right|>0$, so that $\sigma\left(z_{n}\right)=\frac{1}{\left|z_{n}-w_{n}\right|^{1-1 / d}}$. Then $w_{0}=f^{n}\left(w_{n}\right) \in P(f)$, so $\sigma\left(z_{0}\right) \geq \frac{1}{\left|z_{0}-w_{0}\right|^{1-1 / d}}$, and

$$
\frac{\left|\left(f^{n}\right)^{\prime}\left(z_{n}\right)\right| \sigma\left(z_{0}\right)}{\sigma\left(z_{n}\right)} \geq \frac{r_{0}}{r_{n}}\left|\frac{z_{n}-w_{n}}{z_{0}-w_{0}}\right|^{1-1 / d} .
$$


By Koebe's distortion theorem applied to the branch of $f^{-n}$ mapping $U_{0}$ to $U_{n}$, and using (14), we get that

$$
\left|\frac{z_{n}-w_{n}}{z_{0}-w_{0}}\right| \geq \frac{r_{n}}{r_{0}\left(1+\frac{\left|z_{0}-w_{0}\right|}{r_{0}}\right)^{2}} \geq \frac{r_{n}}{4 r_{0}} .
$$

Combining these inequalities, we have that

$$
\begin{aligned}
\frac{\left|\left(f^{n}\right)^{\prime}\left(z_{n}\right)\right| \sigma\left(z_{0}\right)}{\sigma\left(z_{n}\right)} & \geq \frac{r_{0}}{r_{n}}\left(\frac{r_{n}}{4 r_{0}}\right)^{1-1 / d}=\frac{1}{4^{1-1 / d}}\left(\frac{r_{0}}{r_{n}}\right)^{1 / d} \\
& \geq \frac{1}{4^{1-1 / d}}\left(\frac{\epsilon}{2 C_{0} \theta^{n}}\right)^{1 / d} \geq \frac{\epsilon^{1 / d}}{4^{1-1 / d} 2^{1 / d} C_{0}^{1 / d}}\left(\theta^{-1 / d}\right)^{n} \\
& =C_{2} \lambda^{n}
\end{aligned}
$$

Case 3: $f^{n}$ has a critical point in $U_{n}$.

By Theorem 11, there exists $n_{0} \in\{1, \ldots, n\}$ such that $0 \in U_{n_{0}}$, whereas $0 \notin U_{j}$ for $j \in$ $\{1, \ldots, n\} \backslash\left\{n_{0}\right\}$. Furthermore, the theorem guarantees that no backward orbit of $U_{n_{0}}$ contains the critical point 0 , so $U_{j} \cap P(f)=\emptyset$ for $j \geq n_{0}$, and in particular $U_{n} \cap P(f)=\emptyset$. Applying Koebe's $1 / 4$-theorem then gives $\operatorname{dist}\left(z_{n}, P(f)\right) \geq \frac{r_{n}}{4}$. We also know that $w_{0} \in U_{0} \cap P(f)$, so $\operatorname{dist}\left(z_{0}, P(f)\right) \leq\left|z_{0}-w_{0}\right|$. In terms of the metric density $\sigma$, these inequalities are

$$
\sigma\left(z_{n}\right) \leq\left(\frac{4}{r_{n}}\right)^{1-1 / d} \quad \text { and } \quad \sigma\left(z_{0}\right) \geq \frac{1}{\left|z_{0}-w_{0}\right|^{1-1 / d}}
$$

As a further consequence, $\left.f^{n}\right|_{U_{n}}$ is a composition of $n-1$ conformal maps $\left.f\right|_{U_{j}}: U_{j} \rightarrow U_{j-1}$ for $j \neq n_{0}$, and one branched covering $\left.f\right|_{U_{n_{0}}}: U_{n_{0}} \rightarrow U_{n_{0}-1}$ with exactly one branch point at $z=0$. Let $w_{n} \in U_{n}$ be the unique point such that $f^{n-k}\left(w_{n}\right)=0$, and let $w_{0}=f^{k}(0)$. If we endow $U_{0}$ with an orbifold structure by placing a single cone point of index $d$ at $w_{0}$, denoting the resulting orbifold by $\mathcal{O}_{0}$, then $\left.f^{n}\right|_{U_{n}}: U_{n} \rightarrow \mathcal{O}_{0}$ is an orbifold covering map, and thus it is a (local) isometry with respect to the corresponding orbifold metrics $\delta_{U_{n}}$ (which is just the hyperbolic metric of $U_{n}$ ) and $\delta_{\mathcal{O}_{0}}$. Explicitly,

$$
\delta_{U_{n}}(z)=\delta_{\mathcal{O}_{0}}\left(f^{n}(z)\right)\left|\left(f^{n}\right)^{\prime}(z)\right|
$$

for all $z \in U_{n} \backslash\left\{w_{n}\right\}$. Applying this for $z=z_{n}$ and using the relation (3) between conformal radius and the density of the hyperbolic metric, we get

$$
\frac{2}{r_{n}}=\delta_{U_{n}}\left(z_{n}\right)=\delta_{\mathcal{O}_{0}}\left(z_{0}\right)\left|\left(f^{n}\right)^{\prime}\left(z_{n}\right)\right|
$$

Now let $t=p_{U_{0}}\left(z_{0}, w_{0}\right)=\frac{\left|z_{0}-w_{0}\right|}{r_{0}}$ denote the pseudo-hyperbolic distance between $z_{0}$ and $w_{0}$ in $U_{0}=B\left(z_{0}, r_{0}\right)$. Applying Theorem 6 to equation (21) yields

$$
\frac{2}{r_{n}}=\delta_{U_{0}}\left(z_{0}\right) F_{d}(t)\left|\left(f^{n}\right)^{\prime}\left(z_{n}\right)\right| \leq \frac{2\left|\left(f^{n}\right)^{\prime}\left(z_{n}\right)\right|}{r_{0} \cdot t^{1-1 / d}}=\frac{2\left|\left(f^{n}\right)^{\prime}\left(z_{n}\right)\right|}{r_{0}^{1 / d} \cdot\left|z_{0}-w_{0}\right|^{1-1 / d}},
$$

so that

$$
\left|\left(f^{n}\right)^{\prime}\left(z_{n}\right)\right| \geq \frac{r_{0}^{1 / d} \cdot\left|z_{0}-w_{0}\right|^{1-1 / d}}{r_{n}} .
$$


Combining (23) and (19), we get that

$$
\begin{aligned}
\frac{\left|\left(f^{n}\right)^{\prime}\left(z_{n}\right)\right| \sigma\left(z_{0}\right)}{\sigma\left(z_{n}\right)} & \geq \frac{r_{0}^{1 / d}}{r_{n}}\left(\frac{r_{n}}{4}\right)^{1-1 / d}=\frac{r_{0}^{1 / d}}{4^{1-1 / d} \cdot r_{n}^{1 / d}} \\
& \geq \frac{\epsilon^{1 / d}}{4^{1-1 / d} \cdot C_{0}^{1 / d}}\left(\theta^{-1 / d}\right)^{n}=C_{3} \lambda^{n},
\end{aligned}
$$

finishing the proof that $f$ is expanding on $J(f)$ with respect to $\sigma(z)|d z|$.

Theorem 13. Assume that the polynomial $f(z)=z^{d}+c$ is semihyperbolic, but not hyperbolic. Then $f$ is expanding with respect to the metric $\rho(z)|d z|$, where $\rho(z)=1+\frac{1}{\operatorname{dist}(z, P(f))^{1-1 / d}}$. Explicitly, there exist $C>0$ and $\lambda>1$ such that

$$
\left|\left(f^{n}\right)^{\prime}(z)\right| \rho\left(f^{n}(z)\right) \geq C \lambda^{n} \rho(z)
$$

for all $z \in J(f) \backslash P(f)$ and all $n \geq 1$. Furthermore, the metric $\rho(z)|d z|$ is Hölder equivalent to the Euclidean metric on $\mathbb{C}$.

Proof. By Theorem 10, we know that $A_{f}(\infty)$ is a John domain. By definition, semihyperbolic polynomials do not have parabolic periodic points, and from [Mañ93 we know that they do not have Siegel disks either. This shows that any bounded Fatou component for semihyperbolic polynomials is in the basin of some attracting or superattracting periodic cycle, and any such basin contains a critical point. In our situation we have only one critical point, and by the assumption that $f$ is not hyperbolic, it is not contained in an attracting or superattracting basin, which shows that $f$ has no bounded Fatou components, so that the Julia set $J(f)$ is a Gehring tree. By Corollary 9, the metric $\rho(z)|d z|$ is then Hölder equivalent to the Euclidean metric on $\mathbb{C}$. By Theorem 12, $f$ is expanding with respect to $\sigma(z)|d z|$, where $\sigma(z)=\frac{1}{\operatorname{dist}(z, P(f))^{1-1 / d}}$. Since the Julia set $J(f)$ is compact, there exists some $R>0$ such that $J(f) \subseteq B(0, r)$. For $|z| \leq R$ we have that $\sigma(z) \leq \rho(z) \leq(2 R+1) \sigma(z)$, so that

$$
\left|\left(f^{n}\right)^{\prime}(z)\right| \rho\left(f^{n}(z)\right) \geq\left|\left(f^{n}\right)^{\prime}(z)\right| \sigma\left(f^{n}(z)\right) \geq C \lambda^{n} \sigma(z) \geq C^{\prime} \lambda^{n} \rho(z)
$$

with $C^{\prime}=\frac{C}{2 R+1}$, which shows that $f$ is expanding with respect to $\rho(z)|d z|$ as well.

\section{REFERENCES}

[Car97] J. Carette, Liens entre la géométrie et la dynamique des ensembles de Julia, Ph.D. thesis, Université de Paris-Sud (Orsay), 1997, Version available at https: //www. cas. mcmaster. ca/ carette/publications/CaretteThesis. pdf

[CG93] Lennart Carleson and Theodore W. Gamelin, Complex dynamics, Universitext: Tracts in Mathematics, Springer-Verlag, New York, 1993. MR 1230383 (94h:30033)

[CJY94] Lennart Carleson, Peter W. Jones, and Jean-Christophe Yoccoz, Julia and John, Bol. Soc. Brasil. Mat. (N.S.) 25 (1994), no. 1, 1-30. MR 1274760

[Dur83] Peter L. Duren, Univalent functions, Grundlehren der Mathematischen Wissenschaften [Fundamental Principles of Mathematical Sciences], vol. 259, Springer-Verlag, New York, 1983. MR 708494

[GHM89] F. W. Gehring, K. Hag, and O. Martio, Quasihyperbolic geodesics in John domains, Math. Scand. 65 (1989), no. 1, 75-92. MR 1051825

[Joh61] Fritz John, Rotation and strain, Comm. Pure Appl. Math. 14 (1961), 391-413. MR 0138225

[LR18] P. Lin and S. Rohde, Conformal welding of dendrites, Preprint, 2018.

[Mañ93] Ricardo Mañé, On a theorem of Fatou, Bol. Soc. Brasil. Mat. (N.S.) 24 (1993), no. 1, 1-11. MR 1224298

[McM94] Curtis T. McMullen, Complex dynamics and renormalization, Annals of Mathematics Studies, vol. 135, Princeton University Press, Princeton, NJ, 1994. MR 1312365

[Mil06] John Milnor, Dynamics in one complex variable, third ed., Annals of Mathematics Studies, vol. 160, Princeton University Press, Princeton, NJ, 2006. MR 2193309

[MS79] O. Martio and J. Sarvas, Injectivity theorems in plane and space, Ann. Acad. Sci. Fenn. Ser. A I Math. 4 (1979), no. 2, 383-401. MR 565886 
[NV91] Raimo Näkki and Jussi Väisälä, John disks, Exposition. Math. 9 (1991), no. 1, 3-43. MR 1101948

Montana State University, Department of Mathematical Sciences, Bozeman, MT 59717-2400, USA, ORCID ID: HTTPS://ORCID.ORG/0000-0001-5889-9037

E-mail address: geyer@montana.edu 